\title{
Biofertilizantes: alternativa biotecnológica para los agroecosistemas
}

\author{
Biofertilizers: Biotechnological Alternative for Agroecosystems
}

\author{
Mayra Eleonora Beltrán-Pineda ${ }^{a b}$, Andrea Angélica Bernal-Figueroa ${ }^{a c}$ \\ a Grupo de Investigación Gestión Ambiental, Departamento de Biología y Microbiología, Facultad de Ciencias e Ingeniería, \\ Universidad de Boyacá, Colombia \\ b Bióloga. MSc en Ciencias Microbiología. PhD (c) en Biotecnología | mebeltran@uniboyaca.edu.co | https://orcid.org/0000-0002-0451-2535 \\ c Bióloga. MSc en Ingeniería Ambiental | aabernal@uniboyaca.edu.co | https://orcid.org/0000-0001-9024-5666
}

Citation: Beltrán-Pineda, M. E. y BernalFigueroa, A. A. (2022). Biofertilizantes: alternativa biotecnológica para los agroecosistemas. Mutis, 12(1). https://doi.org/10.21789/22561498.1771

Recibido: 16 de junio de 2021 Aceptado: 1 de noviembre de 2021

Copyright: @ 2022 por los autores. Licenciado para Mutis. Este artículo es un artículo de acceso abierto distribuido bajo los términos y condiciones de la licencia Creative Commons Attribution (https:// https://creativecommons.org/licenses/bync-sa/4.0/).

\section{RESUMEN}

El aumento poblacional ha creado una mayor demanda en la producción de alimentos en el ámbito mundial, por lo cual la agricultura es uno de los sectores más representativos, al tiempo que proporciona equilibrio al sistema económico dado su aporte al PIB mundial por la generación de diferentes productos agrícolas. Sin embargo, el desarrollo de prácticas productivas extensivas en busca de mejorar la productividad ha promovido la utilización excesiva de insumos químicos y causado problemas ambientales como eutrofización de fuentes hídricas, acidificación del suelo, disminución de biodiversidad o emisión de gases de efecto invernadero. Por ello, el gran desafío es proporcionar seguridad alimentaria sin afectar la sostenibilidad ambiental. El desarrollo de agricultura sostenible requiere reducción del uso de agroquímicos y reemplazo por productos o procesos más ecológicos, eficientes y económicos; contexto en el que se ha promovido el uso de biofertilizantes teniendo en cuenta la dinámica e importancia de las comunidades microbianas del suelo, así como sus funciones benéficas y cooperativas en la productividad agrícola. Este artículo presenta una revisión acerca del uso de fertilizantes, la contaminación ambiental en agroecosistemas, los biofertilizantes y las propiedades de algunos microorganismos que sirven como base para su fabricación (fijadores de nitrógeno y solubilizadores de fosfato), al igual que los pasos generales del proceso de producción, de tal forma que se promueva el conocimiento de esta biotecnología para manejo de agroecosistemas. Hoy se están produciendo cambios respecto a las prácticas agrícolas y la producción de alimentos en busca de la sostenibilidad; por tal motivo, el estudio de procesos como fijación de nitrógeno molecular y solubilización de fosfatos constituye una base para mejorar la calidad de los insumos biológicos.

Palabras clave: agricultura, fertilizantes, microorganismos fijadores de nitrógeno, microorganismos solubilizadores de fosfatos.

\section{ABSTRACT}

The increase in the world population has generated a greater demand in food production. Therefore, agriculture has become one of its most representative sectors while providing balance to the economic system, given its contribution to world GDP through the generation of different agricultural products. However, the 
development of extensive production practices, which are aimed at improving productivity, has promoted the excessive use of chemical supplies, and caused environmental problems such as eutrophication of water sources, soil acidification, decrease in biodiversity or greenhouse gas emissions. Therefore, providing food security without affecting environmental sustainability is our greatest challenge. The development of sustainable agriculture requires reducing the use of agrochemicals and their replacement by more ecological, efficient, and economical products or processes. In this context, the use of biofertilizers has been promoted considering the dynamics and importance of soil microbial communities, as well as their beneficial and cooperative functions in agricultural productivity. This article presents a review of the use of fertilizers, environmental pollution in agroecosystems, biofertilizers and the properties of some microorganisms that serve as the basis for their manufacture (nitrogen fixers and phosphate solubilizers), as well as the general steps of their production process, in such a way that the knowledge of this biotechnology for agroecosystem management is promoted. Today, changes aimed at improving sustainability are taking place with respect to agricultural practices and food production. For this reason, the study of processes such as molecular nitrogen fixation and phosphate solubilization is the basis for improving the quality of biological inputs.

Keywords: agriculture, fertilizers, nitrogen-fixing microorganisms, phosphatesolubilizing microorganisms.

\section{INTRODUCCIÓN}

En las próximas décadas habrá una presión creciente sobre los sistemas alimentarios mundiales, y la agricultura tendrá el desafío de proporcionar seguridad alimentaria a una población en crecimiento sin afectar la seguridad ambiental (Calabi-Floody et al., 2018). La agricultura es una de las industrias más grandes y proporciona un gran equilibrio al sistema económico, pues un tercio del producto interno bruto (PIB) mundial es aportado por productos agrícolas (Himani y Siddhardha, 2019). De igual forma, el aumento poblacional ha conducido a un incremento en la producción de alimentos, lo cual se consigue si el suelo utilizado para el establecimiento de cultivos de interés agronómico presenta condiciones físicoquímicas y microbiológicas óptimas que permitan sustentar el crecimiento vegetal; no obstante, esto no ocurre en la mayoría de los casos y se hace necesaria la aplicación de agroquímicos.

Actualmente, la calidad de los suelos utilizados con fines agrícolas no es la mejor debido a que las prácticas extensivas utilizan insumos químicos en exceso, lo cual conduce a la erosión de suelos arables, la transformación de ciclos de nutrientes y la disminución de biodiversidad (Chirinos et al., 2006). Los suelos albergan diversas comunidades que apoyan y regulan las funciones del ecosistema, lo cual afecta la producción de plantas y la eficiencia en el uso de los recursos. En este sentido, cada vez hay más estudios que indican cómo la intensificación agrícola afecta la biodiversidad del suelo, y cómo dichos cambios pueden amenazar la seguridad alimentaria actual y futura; de igual forma, se ven comprometidos los servicios que presta el suelo, esenciales para el funcionamiento sostenible de los ecosistemas naturales y gestionados (El Mujtar et al., 2019).

La demanda de productos fertilizantes, así como su producción industrial, ha crecido en las últimas décadas y su consumo es cada vez más habitual en prácticas de agricultura convencional; su utilización ha dado lugar a un fuerte aumento en la producción de alimentos, pero también a graves problemas de contaminación en los 
agroecosistemas (Stefan et al., 2010). Esta problemática ha generado el interés por implementar prácticas de agricultura ecológica que propendan a la conservación del suelo, tales como la rotación de cultivos, el uso de leguminosas de cobertura, la incorporación de enmiendas orgánicas y el uso de poblaciones de microorganismos benéficos que pueden contribuir a largo plazo en la recuperación de la calidad del recurso (Santillana, 2006).

Las poblaciones microbianas son fundamentales para los procesos que impulsan la estabilidad y productividad de los agroecosistemas; en este contexto, varias investigaciones se han realizado para mejorar la comprensión de la diversidad, dinámica e importancia de las comunidades microbianas del suelo, así como sus funciones benéficas y cooperativas en la productividad agrícola (Shankar et al., 2011). El uso de microorganismos en la agricultura es factible debido a su capacidad para descomponer residuos orgánicos, desintoxicar compuestos recalcitrantes, suprimir enfermedades en las plantas, producir compuestos bioactivos como vitaminas y hormonas que estimulan el crecimiento vegetal, $y$ aportar nutrientes al suelo por medio de procesos como la solubilización de fosfato y la fijación de nitrógeno molecular (Çakmakçi et al., 2006).

Los microorganismos fijadores de nitrógeno y solubilizadores de fosfato son utilizados actualmente como base para la fabricación de biofertilizantes, los cuales son insumos biológicos de gran importancia en la agricultura ecológica debido a que promueven la circulación de nutrientes requeridos por las plantas y reducen la necesidad de aplicar fertilizantes químicos. Estos insumos tienen la habilidad de convertir elementos nutricionalmente importantes para las plantas desde su forma no disponible a su forma disponible, a través de procesos biológicos (Vessey, 2003; Alfonso et al., 2005). Se conoce un gran número de hongos y bacterias de vida libre o asociativas que se destacan por su potencial como biofertilizantes; estos forman parte del grupo de los microorganismos promotores de crecimiento vegetal (PGPM, por sus siglas en inglés) y son relevantes para la nutrición de las plantas por cuanto juegan un papel significativo en procesos de biofertilización de cosechas (Çakmakçi et al., 2006).

En este contexto se realizó una revisión bibliográfica en bases de datos como SciELO, ProQuest, Google Scholar, Latindex y ScienceDirect, empleando palabras claves validadas por tesauros especializados, que incluyeron "agroecosistemas", "fertilizantes", "biofertilizantes", "microorganismos fijadores de nitrógeno" y "microorganismos solubilizadores de fosfato". Con esta revisión se pretende dar a conocer algunos aspectos acerca del uso de fertilizantes y su relación con la contaminación ambiental en agroecosistemas, así como abordar qué son los biofertilizantes, las propiedades de algunos microorganismos como base para su fabricación (fijadores de nitrógeno y solubilizadores de fosfato), y los pasos generales en el proceso de producción, de tal forma que se promueva el conocimiento de esta biotecnología para el manejo de agroecosistemas.

\section{Fertilizantes y contaminación ambiental en agroecosistemas}

Los fertilizantes son sustancias de origen mineral producidos ya sea por la industria química o la explotación de yacimientos naturales, utilizados con frecuencia para subsanar las deficiencias nutricionales inherentes a los suelos sobreexplotados (Moreno et al., 2011). Un adecuado suministro de nutrientes es esencial para el crecimiento de los cultivos, pues deben estar disponibles en las cantidades apropia- 
das para que las plantas puedan utilizarlos; sin embargo, debido a las variables fisicoquímicas del suelo y las condiciones climáticas que influencian la toma de estos elementos por parte de las plantas, se hace difícil predecir los niveles de nutrientes ambientalmente seguros que deben aplicarse, y los cultivadores tienden a excederse (Uri, 1997). Los fertilizantes NPK son los más utilizados en la agricultura convencional y aportan tres de los macronutrientes necesarios para el desarrollo de las plantas. La función de cada uno de estos nutrientes se expone en la Tabla 1. Si bien se conocen los beneficios de su aplicación en los suelos, existen reportes de la correlación positiva entre el uso de fertilizantes y algunas enfermedades en las plantas, así como evidencias de que la aplicación de fertilizantes inorgánicos puede suprimir la producción de enzimas del suelo involucradas en los ciclos de nutrientes (De López et al., 1997).

El uso excesivo de fertilizantes químicos en la agricultura causa contaminación ambiental severa sobre los recursos agua, suelo y aire. La presencia de metales pesados y radionucleidos en fertilizantes químicos ha incrementado la amenaza de acumulación de materia inorgánica, eutrofización de fuentes hídricas y, por otra parte, formación de partículas y emisión de gases de efecto invernadero que han contribuido a la pérdida del rendimiento de los cultivos y deterioro de su calidad (Himani y Siddhardha, 2019). De igual forma, la fertilización cambia el número de especies bacterianas en los suelos; altas tasas de nitrógeno pueden reducir el número de bacterias y actinomicetos, y se conoce que se puede generar la recesión de bacterias de los géneros Arthrobacter, Streptomyces, Azotobacter, Rhizobium y Bradyrhzobium (Barabasz et al., 2002).

Tabla 1. Funciones de los principales nutrientes aplicados como fertilizantes

\begin{tabular}{|c|c|}
\hline Nutriente & Función \\
\hline Nitrógeno $(\mathrm{N})$ & $\begin{array}{r}\text { Es componente de los aminoácidos, por lo tanto, es crítico para la formación de proteínas, hace parte de la molé- } \\
\text { cula de clorofila, juega un papel fundamental en la división celular y fotosíntesis. } \\
\text { Su deficiencia puede causar clorosis. }\end{array}$ \\
\hline Fósforo (P) & $\begin{array}{r}\text { Es un elemento esencial para todas las formas de vida debido a que hace parte de los ácidos nucleicos, membra- } \\
\text { nas celulares y del ATP; este debe estar presente en adecuadas cantidades en las células vivas antes de que se dé } \\
\text { la división celular. Es necesario para el crecimiento de la raíz, juega un rol importante en la fotosíntesis, en la } \\
\text { utilización de azúcares y en procesos de transferencia de energía. }\end{array}$ \\
\hline Potasio (K) & $\begin{array}{r}\text { Es esencial para la formación de aminoácidos y proteínas, fotosíntesis, formación de frutos, resistencia de las } \\
\text { plantas a bajas temperaturas y a enfermedades. Es crucial en la translocación de azúcares y otros productos. Se } \\
\text { requiere para mantener la presión interna de la planta y prevenir la marchitez. }\end{array}$ \\
\hline
\end{tabular}

Fuente: Uri (1997).

En agroecosistemas, la aplicación desmedida de fertilizantes NPK fomenta la acidificación del suelo, la destrucción de hábitats naturales con bajo contenido en nutrientes y causa eutrofización de las aguas (Himani y Siddhardha, 2019). Se observan con preocupación los efectos nocivos del exceso de nitrógeno y fósforo sobre el medio ambiente; por ejemplo, se conoce que el nitrógeno se moviliza hacia aguas superficiales y refleja mucho más el efecto de la actividad agrícola sobre el ambiente acuático que el fósforo, aunque este último se une al sedimento, lo cual erosiona el terreno (Smith et al., 1987). Se estima que del $15 \%$ al $50 \%$ de los fertilizantes nitrogenados son lixiviados a las capas profundas del suelo y a corrientes de aguas superficiales, favoreciendo la eutrofización (Barabasz et al., 2002). Este fenómeno se define como un proceso de deterioro de la calidad del recurso agua originado por el enriquecimiento de nutrientes, principalmente nitrógeno en forma de sales de nitrato o amonio, y fósforo en forma de fosfatos, condicionando 
la utilización de estos y ejerciendo grandes impactos ecológicos, sanitarios y económicos a escala regional (Ledesma et al., 2013).

El aumento de nutrientes en los cuerpos hídricos estimula el crecimiento de algas, lo cual provoca un enturbiamiento que impide la penetración de la luz hasta las profundidades del ecosistema, se disminuye la tasa de fotosíntesis y, por lo tanto, la producción de oxígeno libre también disminuye; el fondo del ecosistema acuático se va convirtiendo de forma gradual en un ambiente anaerobio, debido al aumento en la concentración de gases como sulfuro de hidrógeno $\left(\mathrm{H}_{2} \mathrm{~S}\right)$, dióxido de carbono $\left(\mathrm{CO}_{2}\right)$ y metano $\left(\mathrm{CH}_{4}\right)$, lo cual reduce en gran medida la biodiversidad y hace que el cuerpo hídrico no sea apto para el desarrollo de la vida (Uri, 1997). Por otra parte, debido a este proceso, se incrementa el crecimiento de muchas especies de fitoplancton, incluidas cianófitas, que son peligrosas para los seres humanos debido a que causan enfermedades gastrointestinales e incluso parálisis; en animales pueden causar serios problemas de envenenamiento (Chen et al., 2006).

Asimismo, la contaminación por nitratos es un problema en muchos cuerpos de agua superficiales y subterráneos que están hidráulicamente conectados con campos agrícolas (Kellman y Hillaire-Marcel, 2003); esto ocurre por cuanto una vez son aplicados los fertilizantes nitrogenados, gran parte son asimilados por las plantas debido a su solubilidad, pero si hay un exceso, el nitrógeno que no se utiliza puede ser inmovilizado, desnitrificado, escurrido a aguas superficiales, causando diversos efectos biológicos ya mencionados, o lixiviado desde la zona radicular a través del suelo a las aguas subterráneas (Kundu et al., 2009).

Se conoce que aguas con altas concentraciones de nitratos muchas veces se utilizan para el consumo humano y se han evidenciado problemas de salud como la metahemoglobinemia (síndrome del bebe azul) en niños, y cáncer de estómago en adultos (Kundu et al., 2009). Por otra parte, altas tasas de fertilización nitroge-

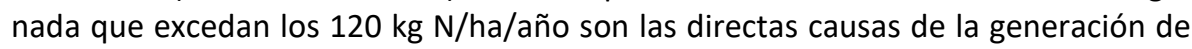
nitrosaminas, sustancias carcinogénicas, fitotóxicas y mutagénicas que pueden tener consecuencias ecológicas graves y efectos sobre los micro- y macroorganismos; asimismo, son inhibidoras de la síntesis de ADN y ARN y bloquean la transmisión de información genética a nivel de síntesis de aminoácidos y formación de ciertas fitohormonas tales como el ácido naftal-acético (NAA), responsable de la rizogénesis en plantas superiores (Barabasz et al., 2002).

Nziguheba y Smolders (2008) indican que algunas trazas de metales pesados pueden llegar a los suelos agrícolas gracias a los fertilizantes minerales y, por lo tanto, constituyen un riesgo potencial para el ambiente y la salud humana. Los fertilizantes fosforados se derivan principalmente de la roca fosfórica y, de acuerdo con su origen, pueden contener trazas de elementos menores; se han registrado altas concentraciones de uranio en la roca fosfórica, elemento de larga vida que se conoce por su radiotoxicidad y su toxicidad química (Takeda et al., 2006). Los niveles de uranio en suelos agrícolas pueden ser elevados por los largos periodos de aplicación de fertilizantes fosforados; sin embargo, su grado de acumulación puede verse afectado por factores como el tipo de suelo, la tasa de aplicación y la calidad de los fertilizantes (Takeda et al., 2006); por lo tanto, cuando estos elementos son aplicados a los suelos, pueden persistir durante largo tiempo y volverse disponibles para las plantas, especialmente en suelos ácidos, con el riesgo potencial de bioacumulación y efectos a lo largo de la cadena alimenticia (De López et al., 1997). 
Además de los problemas ambientales y de salud humana generados por el uso desmedido de fertilizantes, la agricultura ha encontrado problemas en su rentabilidad debido a la baja eficiencia de la fertilización y los altos costos de dichos productos requeridos para subsanar los problemas físicoquímicos del suelo (Uri, 1997). En consecuencia, será necesario utilizar tecnologías modernas en los agroecosistemas para suministrar alimentos suficientes y disminuir los impactos negativos sobre el medio ambiente, inducidos por la fertilización química y la eliminación o reutilización inadecuada de los desechos agrícolas (Calabi-Floody et al., 2018). Por tal motivo, se hace necesaria la implementación de prácticas que limiten la aplicación de insumos de tipo sintético, y es en este contexto donde los biofertilizantes se convierten en una alternativa viable e importante para lograr un desarrollo agrícola ecológicamente sostenible.

\section{Biofertilizantes como alternativa para la agricultura sostenible}

El desarrollo de la agricultura sostenible requiere de una fuerte reducción de agroquímicos y su reemplazo por productos más ecológicos, eficientes y económicos; y aunque existen técnicas únicas para cultivos específicos, las prácticas comúnmente utilizadas incluyen rotación, cultivos sin labranza, manejo integrado de plagas, abonos verdes, policultivo y uso de los llamados biofertilizantes (Piotrowski y Rillig, 2008). En el ámbito mundial, la aplicación de microorganismos benéficos en prácticas agrícolas comenzó hace más de sesenta años, práctica que ha sido objeto de investigaciones intensivas durante las últimas décadas. Existe un incremento en la evidencia de que las poblaciones microbianas benéficas pueden aumentar la resistencia de las plantas a condiciones medioambientales adversas como la deficiencia de nutrientes (Aguilar, 2007).

En este sentido, la biotecnología ofrece herramientas para cumplir con este cometido cuando se integra debidamente con otras tecnologías. Un biofertilizante se define como una sustancia que contiene microorganismos benéficos del suelo, a menudo mutualistas, que pueden promover el crecimiento de las plantas y reducir el uso de insumos, los cuales pueden aplicarse a las semillas, a las superficies de las plantas o al suelo, y pueden colonizar la rizósfera o el interior de estas, impulsando así el crecimiento vegetal (Vessey, 2003; Piotrowski y Rillig, 2008; Bhattacharyya y Jha, 2012; Verzeaux et al., 2017).

Estas sustancias se producen a partir de microorganismos que viven normalmente en el suelo, aunque en bajas densidades (Aguilar, 2007), los cuales cumplen su función con un mínimo uso de recursos no renovables; además tienen la ventaja de que los procesos microbianos son rápidos, y los biopreparados pueden aplicarse en pequeñas unidades agrícolas para solucionar problemas locales específicos (Castilla, 2006). Se ha demostrado que las plantas inoculadas con algún microorganismo que estimule su crecimiento y desarrollo presentan una mayor capacidad para absorber más eficientemente el agua y los nutrientes del suelo, a través del estímulo provocado en el sistema radical que se evidencia en el estado nutricional de las plantas (Alfonso et al., 2005).

De igual forma, se conoce que el uso de biofertilizantes permite una producción a bajo costo, no contamina el ambiente, mantiene la conservación del suelo desde el punto de vista de fertilidad y biodiversidad, además representa una importante alternativa para mejorar la productividad de los cultivos y facilitar la recuperación de terrenos marginales para su aprovechamiento agrícola y forestal 
(Alfonso et al., 2005; Chirinos et al., 2006). Los biofertilizantes podrían contribuir en gran medida a satisfacer las necesidades de alimentos de una población en crecimiento (Delgadillo et al., 2001). Dichos microorganismos pueden comprender poblaciones mixtas de microbios naturales que se pueden aplicar como inoculantes para aumentar la diversidad microbiana del suelo; las investigaciones han demostrado que la inoculación de una comunidad microbiana eficiente mejora la salud y calidad del suelo y, por ende, el crecimiento, rendimiento y calidad de los cultivos. Estas poblaciones pueden consistir en especies seleccionadas de microorganismos que incluyen rizobacterias promotoras del crecimiento de las plantas, cianobacterias fijadoras de $\mathrm{N}_{2}$, bacterias y hongos supresores de enfermedades de las plantas, microbios que degradan los tóxicos del suelo, actinomicetos y otros microbios útiles (Shankar et al., 2011).

\section{Microorganismos promotores de crecimiento vegetal (PGPM) como biofertilizantes}

Las especies bacterianas o fúngicas del suelo que imparten algún beneficio a las plantas se conocen comúnmente como microorganismos promotores del crecimiento vegetal (PGPM, por sus siglas en inglés), muchas de las cuales se han desarrollado para su distribución comercial como enmiendas del suelo para implementación en sistemas agrícolas (Lapsansky et al., 2016). Este grupo está representado por numerosas especies bacterianas y fúngicas incluidas las micorrizas, que están asociadas con la mayoría de especies vegetales y comúnmente se les encuentra en la mayoría de los ambientes (Caballero, 2006). Los microorganismos del suelo interactúan específicamente con las raíces de las plantas en la rizósfera, donde su número es generalmente mayor que en el suelo libre debido a que se allí se establece una relación simbiótica planta-microorganismo (Kloepper et al., 1989). El término propuesto específicamente para las rizobacterias que cuentan con la capacidad de promover el crecimiento de plantas es PGPR, o Promoting Growth Plant Rhizobacteria, que han sido ampliamente estudiadas por tener propiedades biofertilizantes, aunque también se destacan especies fúngicas con esta capacidad. El uso de estos microorganismos como biofertilizantes es una biotecnología promisoria para incrementar la producción primaria con menos cantidad de fertilizantes, además de ser una tecnología ecoamigable (Compant et al., 2005).

Algunos de los microorganismos utilizados como base para la producción de biofertilizantes forman parte del grupo de los PGPM; sin embargo, no todos son biofertilizantes, muchos de estos microorganismos estimulan el crecimiento de la planta por otros mecanismos (Vessey, 2003). De acuerdo con su efecto en la promoción de crecimiento vegetal, los PGPM pueden ser clasificados en promotores directos, que son los microorganismos productores de hormonas de crecimiento vegetal, y los promotores de crecimiento indirectos, donde se encuentran los microorganismos con potencial biofertilizante, aquí se incluyen los fijadores biológicos de nitrógeno de vida libre o que forman nódulos radiculares (simbióticos), así como los microorganismos que incrementan la disponibilidad de nutrientes en la rizósfera como los solubilizadores de fosfatos (Caballero, 2006; Bhattacharyya y Jha, 2012).

\section{Microorganismos fijadores de nitrógeno}

El nitrógeno es el componente principal de los aminoácidos y ácidos nucleicos, sustancias fundamentales para los seres vivos (Atlas y Bartha, 2002); su deficiencia en las plantas detiene su desarrollo y se manifiesta principalmente co- 
mo una clorosis generalizada, especialmente en las hojas más senescentes. La mayoría de los suelos del mundo son deficientes en nitrógeno y la aplicación de fertilización nitrogenada es esencial para mantener los buenos rendimientos; generalmente la urea es la fuente de nitrógeno más conveniente, pero desafortunadamente solo cerca del $50 \%$ de la aplicada es tomada por la planta; esta baja eficiencia se debe a la volatilización de amonio, la desnitrificación y las pérdidas por lixiviación (Sánchez et al., 2006).

El nitrógeno se encuentra en forma gaseosa en el aire atmosférico, y las plantas y animales no pueden utilizarlo en esa forma para su metabolismo (Dobereiner et al., 1995). Las plantas toman el nitrógeno principalmente a través de fertilizantes nitrogenados que son industrialmente sintetizados a partir de dinitrógeno atmosférico $\left(\mathrm{N}_{2}\right)$, elemento que puede hacerse disponible para las plantas gracias a la fijación biológica de nitrógeno (FBN), el cual, después de la fotosíntesis, es considerado el más importante del planeta (Sánchez et al., 2006). Este proceso es realizado por ciertas bacterias denominadas diazotróficas, las cuales poseen el sistema enzimático para romper el triple enlace entre los dos átomos de nitrógeno que forman el dinitrógeno atmosférico, y transformarlo en dos moléculas de amonio, proceso similar al que ocurre a nivel industrial durante la fabricación de fertilizantes nitrogenados (Kanimozhi y Panneerselvam, 2010). El complejo enzimático responsable se denomina nitrogenasa y consta de dos proteínas: la dinitrogenasa y la dinitrogenasa reductasa, cada una con múltiples subunidades; el complejo tiene elementos como hierro, molibdeno, vanadio y requiere de iones Mg+ para ser activado, es sensible al oxígeno y necesita mucha energía para su funcionamiento debido a que se requieren 16 moléculas de ATP por cada dos moléculas de $\mathrm{NH}_{3}$ producidas (Sánchez et al., 2006).

La fijación de nitrógeno es un proceso altamente regulado, limitado por la fuente de carbono aprovechable, y se ve inducido por bajos niveles de nitrógeno combinado; se encuentra que los microorganismos evitan el alto costo de síntesis y operación enzimática cuando existe suficiente nitrógeno disponible (Sttenhoudt y Vanderleyden, 2000). Los microorganismos fijadores de este elemento pueden realizar el proceso de fijación de manera asimbiótica, llevada a cabo por bacterias de vida libre, o simbiótica, efectuada por bacterias que forman nódulos radiculares. En la Tabla 2 se pueden evidenciar los principales géneros de bacterias fijadoras de nitrógeno.

La fijación simbiótica de nitrógeno se limita solo a leguminosas y algunos árboles o arbustos que se asocian con Frankia sp. (Bhattacharyya y Jha, 2012). En hábitats terrestres, esta fijación biológica llevada a cabo por los rizobios representa la mayor contribución de nitrógeno combinado; esta suele ser de dos a tres órdenes de magnitud superior a la tasa de fijación de las bacterias fijadoras de nitrógeno de vida libre en el suelo y en ambientes acuáticos. Las cianobacterias son los principales organismos fijadores de nitrógeno y su tasa de fijación suele ser uno o dos órdenes de magnitud superior a la tasa de fijación de las bacterias no fotosintéticas libres del suelo (Atlas y Bartha, 2002).

La posibilidad de utilizar microorganismos del suelo que favorezcan la nutrición y desarrollo de las plantas ofrece nuevas alternativas para incrementar el rendimiento y mejorar la eficiencia del uso de fertilizantes minerales. Algunos de estos microorganismos se vinculan con la fijación biológica de nitrógeno e incorporan al suelo cantidades variables de dicho elemento (Alfonso et al., 2005). Dentro de las metodologías aplicadas para la determinación de fijación biológica de nitró- 
geno, generalmente se aíslan bacterias con esta capacidad, y se utilizan medios de cultivo libres o desprovistos de nitrógeno de tal forma que la bacteria que crece allí lo hace gracias a su capacidad de fijar el nitrógeno atmosférico. Se destaca el medio de cultivo ASHBY para el aislamiento selectivo de Azotobacter sp., pero en general se pueden utilizar Winogradsky y NFB (Dobereiner et al., 1995).

Tabla 2. Principales géneros de bacterias fijadoras de nitrógeno.

\begin{tabular}{|c|c|c|}
\hline Característica & \multicolumn{2}{|c|}{ Géneros } \\
\hline Simbióticas & Rhizobium sp. & Bradyrhizobium sp. \\
\hline \multirow{11}{*}{ De vida libre } & Azotobacter sp. & Bacillus sp. \\
\hline & Beijerinckia sp. & Azoarcus sp.( endofítico) \\
\hline & Klebsiella sp. & Pantoea sp. \\
\hline & Burkholderia sp.(endofítico) & Azospirillum sp. \\
\hline & Gluconacetobacter sp. (endofítico) & Pseudomonas sp. \\
\hline & Chromatium sp. & Rhodopseodomonas sp. \\
\hline & Rhodospiriilum sp. & Rhodomicrobium sp. \\
\hline & Chlorobium sp. & Desulfovibrio sp. \\
\hline & Desulfotomaculum sp. & Klebsiella sp. \\
\hline & Clostridium sp. & Vibrio sp. \\
\hline & Thiobacillus sp. & Herbaspirillum sp. (endofítico) \\
\hline \multirow{6}{*}{ Cianobacterias } & Anabaena sp. & Aphanizomenon sp. \\
\hline & Nostoc sp. & Gloeotrichia sp. \\
\hline & Cylindrospermum sp. & Calothrix sp. \\
\hline & Scytonema sp. & Scytonema sp. \\
\hline & Oscillatoria sp. & Trichodesmium sp. \\
\hline & Microcoleus sp. & Lyngbya sp. \\
\hline
\end{tabular}

Fuente: Hurek et al. (1994), Baldani et al. (1997), Antoun et al. (1998), Riggs et al. (2001), Yanni et al. (2001), Atlas y Bartha (2002), Vessey (2003) y Bhattacharyya y Jha (2012).

Los estudios de tasas de fijación de nitrógeno avanzaron mucho al desarrollarse las pruebas de reducción de acetileno, las cuales se basan en que la enzima nitrogenasa reduce el acetileno a etileno; el parecido entre la molécula de acetileno $(\mathrm{HC}-\mathrm{CH})$ y nitrógeno $(\mathrm{N}-\mathrm{N})$ es evidente, y de este modo la tasa de formación de etileno es también una medida de actividad fijadora de nitrógeno o nitrogenasa; el etileno puede medirse fácilmente con un cromatógrafo de gases equipado con un detector de ionización (Atlas y Bartha, 2002). De igual forma, se puede usar un análisis micro Kjeldahl para determinar la eficiencia de fijación de nitrógeno (Kanimozhi y Panneerselvam, 2010).

\section{Microorganismos solubilizadores de fosfato}

El fósforo es un elemento esencial para todos los organismos vivos, por formar parte de los ácidos nucleicos, ATP y fosfolípidos, componentes fundamentales de la membrana plasmática (Atlas y Bartha, 2002). Su principal función fisiológica es intervenir en procesos de acumulación y liberación de energía durante el metabolismo celular (Coyne, 2000). El fósforo es el segundo nutriente mineral, después del nitrógeno, que limita el crecimiento de las plantas terrestres $\mathrm{y}$, por tanto, la producción agropecuaria; irónicamente los suelos tienen grandes reservas de este elemento, pero las cantidades disponibles para las plantas son una escasa proporción del total existente (Vessey, 2003); su baja disponibilidad se debe a que la mayor parte está presente en formas insolubles y las plantas solo pueden absorber dos formas solubles del mineral: el ión monobásico $\left(\mathrm{H}_{2} \mathrm{PO}_{4}{ }^{-}\right)$y el ión dibásico $\left(\mathrm{HPO}_{4}{ }^{2-}\right)$ (Kim et al., 1998). 
Se conoce que su disponibilidad está restringida por su tendencia a precipitarse en presencia de metales bivalentes $\left(\mathrm{Ca}^{2+}, \mathrm{Mg}^{2+}\right)$ y el ión férrico $\left(\mathrm{Fe}^{3+}\right)$ a $\mathrm{pH}$ neutro $O$ alcalino, transformándose a formas insolubles de $P$ (Bhattacharyya y Jha, 2012). Los microorganismos solubilizadores de fosfato son importantes en los suelos debido a que pueden transformar fosfato inorgánico insoluble en ortofosfato, que es una forma de $\mathrm{P}$ asimilable para las plantas, siendo componentes importantes en su ciclo edáfico; por esta razón, también pueden ser utilizados para la fabricación de fertilizantes.

Los microorganismos solubilizadores de fosfato (MSF) están presentes en los suelos y son comunes en la rizósfera, constituyen un grupo importante de los promotores de crecimiento vegetal o PGPM (Rosas et al., 2006), y establecen una relación simbiótica con la planta en la que el microorganismo moviliza el fosfato insoluble presente en el suelo para que pueda ser tomado por la raíz, mientras que la planta le suministra compuestos carbonados que son metabolizados para el crecimiento microbiano (Pérez et al., 2007). Muchas bacterias solubilizan rocas y minerales esenciales para el crecimiento vegetal a través de la producción de numerosos ácidos orgánicos y enzimas fosfatasas (Kim et al., 1998; Caballero, 2006), pero el mecanismo de solubilización más estudiado es la acidificación del medio extracelular por la secreción de ácidos orgánicos, tales como el ácido glucónico o cetoglucónico (Bhattacharyya y Jha, 2012; Beltrán, 2014). Se conoce que algunos microorganismos quimiolitótrofos como Nitrosomona sp. y Thiobacillus sp. movilizan fosfatos inorgánicos y producen ácido nitroso y sulfúrico, respectivamente (Tiessen y Stewart, 1985).

Son diversos los grupos microbianos capaces de solubilizar el fósforo edáfico, y entre ellos los de mayor relevancia son los hongos, muchos de los cuales son patógenos como Aspergillus sp., Fusarium sp. o Sclerotium sp.; un segundo grupo lo constituyen los actinomicetos productores de sustancias antibióticas, y existen también varios géneros bacterianos reconocidos, entre los que se pueden mencionar Bacillus sp., Flavobacterium sp., Pseudomonas sp., Mycobacterium sp., Micrococcus sp., Rhizobium sp., Mesorhizobium sp. y Sinorhizobium sp. (Rosas et al., 2006). En la Tabla 3 se pueden evidenciar los principales géneros de microorganismos solubilizadores de fosfato.

Para el estudio de microorganismos solubilizadores, inicialmente se deben aislar del suelo o la rizósfera, a través del método de diluciones seriadas con siembra en medios de cultivo selectivos que contengan una fuente de fosfato insoluble; dentro de los medios más utilizados se encuentran el Pikovskays's (Pikovskaya, 1948), SRS (Sundara y Sinha, 1963) y NBRIP (Nautiyal, 1999), donde se evidenciarán halos de solubilización o acidificación según el medio de cultivo empleado como criterio inicial de selección. Posteriormente, los aislamientos son sometidos a una evaluación cuantitativa de la actividad en cultivo líquido utilizando pruebas colorimétricas para la detección de fosfatos solubles, las cuales tienen como principio el método del azul molibdeno sugerido por Chen et al. (2006), un procedimiento análogo al us Standart methods 4500-P (APHA, 2002). Cuando se han logrado seleccionar cepas con potencial solubilizador de fosfato a nivel in vitro, se debe evaluar su efectividad de promoción de crecimiento vegetal a nivel invernadero y campo (Legget y Steckler, 2007). 
Tabla 3. Principales géneros de microorganismos solubilizadores de fosfato

\begin{tabular}{|c|c|c|}
\hline Tipo de microorganismo & \multicolumn{2}{|c|}{ Géneros } \\
\hline \multirow{17}{*}{ Bacterias } & Achromobacter sp. & Acinetobacter sp. \\
\hline & Aereobacter sp. & Agrobacterium sp. \\
\hline & Arthrobacter sp. & Azotobacter sp. \\
\hline & Bacillus sp. & Bradyrhizobium sp. \\
\hline & Burkholderia sp. & Chryseobacterium sp. \\
\hline & Delftia sp. & Enterobacter sp. \\
\hline & Erwinia sp. & Flavobacterium sp. \\
\hline & Gordonia sp. & Kitasatospora sp. \\
\hline & Klebsiella sp. & Mesorhizobium sp. \\
\hline & Micrococcus sp. & Mycobacterium sp. \\
\hline & Pantoea sp. & Phyllobacterium sp. \\
\hline & Pseudomonas sp. & Rahnella sp. \\
\hline & Ralstonia sp. & Rhizobium sp. \\
\hline & Rhodobacter sp. & Rhodococcus sp. \\
\hline & Serratia sp. & Sinorhizobium sp. \\
\hline & Streptomyces sp. & Streptosporangium sp. \\
\hline & Thiobacillus sp. & Yarowia sp. \\
\hline \multirow{6}{*}{ Hongos } & Aspergillus sp. & Fusarium sp. \\
\hline & Mucor sp. & Paecillomyces sp. \\
\hline & Penicillium sp. & Rhizopus sp. \\
\hline & Sclerotium sp. & Scopulariopsis sp. \\
\hline & Sytalidium sp. & Talaromyces sp. \\
\hline & Trichocladium sp. & \\
\hline
\end{tabular}

Fuente: Antoun et al. (1998), Chabot et al. (1998), Kumar y Narula (1999) y Beltrán (2014).

La inoculación del suelo o las semillas con bacterias solubilizadoras de fosfato mejora la solubilización del fósforo fijado en el suelo, lo cual resulta en cultivos con mayores rendimientos. Asimismo, los biofertilizantes a base de bacterias solubilizadoras de fosfato son considerados entre los más efectivos insumos para suministrar fósforo a niveles aceptables; la aplicación de biofertilizantes promueve el incremento en la población de microorganismos efectivos y la actividad microbiana alrededor de la raíz, lo cual aumenta la habilidad de la planta para absorber nutrientes (Mehrvarz y Chaichi, 2008). Es importante considerar también que el crecimiento de bacterias solubilizadoras de fosfato depende de actividades culturales y diferentes propiedades del suelo, tanto físicas como químicas, donde se destacan el contenido de materia orgánica y fósforo en el sistema (Kim et al., 1998).

\section{Producción de biofertilizantes a nivel industrial}

El uso de inoculantes microbianos incluye la selección y multiplicación de microorganismos benéficos para las plantas, tanto de aquellos que la protegen contra el ataque de patógenos, plagas y malezas, como de aquellos que proporcionan nutrimentos (Delgadillo et al., 2001). Gracias a estos grupos microbianos se pueden fabricar los biofertilizantes; en la actualidad existe un creciente interés en el uso de microorganismos con estos fines, por lo cual son indispensables los estudios básicos y aplicados hacia la generación de tecnologías que permitan la utilización masiva de este tipo de biopreparados microbianos en cultivos de interés agronómico (Valero, 2003). 
La producción de inoculantes microbianos es un proceso complejo que requiere varias etapas y su objetivo es generar un producto seguro, efectivo, fácil de usar y con precios asequibles para los productores. Este proceso inicia con la obtención de microorganismos, los cuales pueden provenir de colecciones mundiales, de otros investigadores o del ambiente; en este último caso se debe realizar el proceso de aislamiento a partir de muestras de suelo total o de zonas rizosféricas, y siembra en medios de cultivo selectivos para que se pueda evidenciar la actividad de interés. Se deben realizar evaluaciones cualitativas y cuantitativas como criterio de selección, y cuando se tienen los microorganismos potenciales en cultivo puro, es necesario evaluar su estabilidad funcional, pues algunos organismos pierden su habilidad después de ser aislados, y tras subcultivos sucesivos, de igual forma, se requiere garantizar la inocuidad de la cepa seleccionada (Legget y Steckler, 2007).

Adicionalmente, deben realizarse pruebas de efectividad a nivel invernadero y campo en las que se evalúe que los microorganismos puedan promover el crecimiento vegetal y sean buenos competidores frente a otros microorganismos de la rizósfera, teniendo en cuenta que el suelo rara vez provee un ambiente rico en nutrientes como los medios de cultivo. Otro aspecto por considerar es que muestren adaptabilidad para ser efectivos y eficientes sobre un rango de condiciones geográficas y ambientales limitantes (Stephens y Rask, 2000).

Posteriormente, se formulan medios de cultivo de acuerdo con el microorganismo y se realizan procesos de fermentación a pequeña y gran escala, donde se controlan parámetros como la concentración de nutrientes, oxígeno disuelto, temperatura, $\mathrm{pH}$ y velocidad de agitación. La última etapa del proceso es la formulación del inoculante, donde se selecciona el soporte adecuado y se define su presentación con su respectivo control de calidad (Stephens y Rask, 2000). Los inoculantes son comercializados en tres presentaciones: polvo, líquido o granular. Los productos en polvo son usados especialmente para la inoculación de semillas; los granulares pueden colocarse directamente en el surco o por debajo de la semilla; y los líquidos pueden ser aplicados directamente en el surco en el momento de la siembra, están disponibles en diferentes formulaciones de caldo y son los más fáciles de manejar (Hynes et al., 1995).

Los principales problemas en la producción de inoculantes son la calidad y tratamiento del soporte (carrier), la pureza y eficiencia del cultivo, y la consecución del número de células viables en el producto final (Stephens y Rask, 2000), teniendo en cuenta que el soporte utilizado debe tener dos propiedades fundamentales: soportar el crecimiento del organismo modelo (planta) y mantener viables las poblaciones microbianas deseables por un periodo aceptable. En general, se puede concluir que lo que hace a un microorganismo un buen bioinoculante es que sea fácil de producir y formular, funcionalmente estable, seguro, competitivo en la rizósfera, y efectivo bajo un amplio rango de condiciones en campo (Legget y Steckler, 2007). Los PGPM que se introducen en los suelos deben poder colonizar el área alrededor (rizósfera) o directamente dentro (endofitos) de las raíces, competir con otros microbios por recursos limitados, y persistir en el ambiente del suelo; por lo tanto, mejorar los suelos con la aplicación de microbios beneficiosos produce resultados inherentemente variables, ya que las bacterias pueden ser impredecibles en términos de establecimiento y grado de promoción del crecimiento de las plantas (Lapsansky et al., 2016). 


\section{Conclusiones}

Los problemas ambientales a causa del uso desmedido de fertilizantes sintéticos en los campos agrícolas han generado preocupación en la comunidad científica en general, que ha trabajado en el desarrollo de estrategias biotecnológicas para subsanar tal problemática. En consecuencia, el desarrollo de biofertilizantes se ha convertido en una alternativa que contribuye a la sostenibilidad ambiental y productividad de los cultivos, debido al papel fundamental de las poblaciones microbianas durante los procesos que promueven la estabilidad y productividad de los agroecosistemas.

Se están produciendo cambios respecto a las prácticas agrícolas y producción de alimentos en busca de la sostenibilidad; por tal motivo, el estudio de procesos como la fijación de nitrógeno molecular y la solubilización de fosfatos constituye una base para mejorar la calidad de los insumos biológicos, los cuales pueden ser un medio eficaz y potencial para mejorar la fertilidad del suelo y el rendimiento de los cultivos.

\section{Referencias}

Aguilar, N. (2007). Evaluación del efecto de la inoculación de rizobacterias promotoras del crecimiento vegetal y solubilizadoras de fósforo en plantas de trigo (Triticum aestivum L.). Santiago de Chile: Instituto de Producción y Sanidad Vegetal.

Alfonso, E., Leyva, A. y Hernández, A. (2005). Microorganismos benéficos como biofertilizantes eficientes para el cultivo del tomate (Lycopersicon esculentum, Mill). Rev. Colomb. Biotecnol, 2(2), 47-54.

Antoun, H., Beauchamp, C., Goussard, N., Chabot, R. y Lalande, R. (1998). Potential of Rhizobium and Bradyrhizobium species as plant growth promoting rhizobacteria on non-legumes: effect on radishes (Raphanus sativus L.). Plant Soil, 204, 57-67. https://doi.org/10.1023/A:1004326910584

APHA (2002). Standard Methods for the Examination of Water and Wastewater. Washington: American Public Health Association.

Atlas, R. y Bartha, R. (2002). Ecología microbiana y microbiología ambiental. Madrid: Pearson.

Baldani, J., Caruso, L., Baldani, V., Goi, S. y Döbereiner, J. (1997). Recent advances in BNF with non-legume plants. Soil Biology and Biochemistry, 29(5-6), 911-922. https://doi.org/10.1016/S0038-0717(96)00218-0

Barabasz, W., Albińska, D., Jaśkowska, M. y Lipiec, J. (2002) Biological effects of mineral nitrogen fertilization on soil microorganisms. Polish Journal of Environmental Studies, 11(3), 193-198.

Beltrán, M. (2014). La solubilización de fosfatos como estrategia microbiana para promover el crecimiento vegetal. Corpoica Cienc. Tecnol. Agropecu, 15(1), 101-113. https://doi.org/10.21930/rcta.vol15_num1_art:401 
Bhattacharyya, P. y Jha, D. (2012) Plant growth-promoting-rhizobacteria (PGPR): emergence in agriculture. World J. Microbiol Biotechnol., 28, 1327-1350. https://doi.org/10.1007/s11274-011-0979-9

Caballero, J. (2006). Microbiología agrícola e interacciones microbianas con plantas. Revista Latinoamericana de Microbiología, 48(2), 154-161.

Çakmakçi, R., Dönmez, F., Aydın, A. y Şahin, F. (2006). Growth promotion of plants by plant growth-promoting rhizobacteria under greenhouse and two different field soil conditions. Soil Biol. Biochem., 38(6), 1482-1487. https://doi.org/10.1016/j.soilbio.2005.09.019

Calabi-Floody, M., Medina, J., Rumpel, C., Condron, L., Hernández, M., Dumont, M. y De la Luz, M. (2018). Smart Fertilizers as a Strategy for Sustainable Agriculture. Advances in Agronomy, 147, 119-157. https://doi.org/10.1016/bs.agron.2017.10.003

Castilla, L. (2006). Fertilización bio-orgánica en el cultivo del arroz. En L. Castilla y M. Bolaños (Eds.), La biofertilización: una alternativa viable para la nutrición vegetal. Ibagué: Sociedad Colombiana de la Ciencia del Suelo.

Chabot, R., Beauchamp, C., Kloepper, J. y Antoun, H. (1998). Effect of phosphorus on root colonization and growth promotion of maize by bioluminescent mutants of phosphate-solubilizing Rhizobium leguminosarum biovar phaseoli. Soil Biol. Biochem., 30(12), 1615-1618. https://doi.org/10.1016/S00380717(98)00054-6

Chen, Y., Rekha, P., Arun, A., Shen, F., Lai, W. y Young, C. (2006). Phosphate solubilizing bacteria from subtropical soil and their tricalcium phosphate solubilizing abilities. Appl. Soil. Ecol., 34(1), 33- 41. https://doi.org/10.1016/j.apsoil.2005.12.002

Chirinos, J., Leal, A. y Montilla, J. (2006). Uso de insumos biológicos como alternativa para la agricultura sostenible en la zona sur del estado Anzoátegui. $\begin{array}{llll}\text { Revista Digital Hoy, } & 11 .\end{array}$ https://ingenieriadereacciones.files.wordpress.com/2013/02/5-zona-sur-deanzoatgui-bioprodctos.pdf

Compant, S., Brion, D., Jerzy, N., Christophe, C. y Essaïd, A. (2005). Use of plant growth-promoting bacteria for biocontrol of plant diseases: principles, mechanisms of action and future prospects. Appl. Environ. Microbiol., 71(9), 4951-4959. https://doi.org/10.1128/AEM.71.9.4951-4959.2005 drid: Paraninfo.

Coyne, M (2000). Microbiología del suelo. Un enfoque exploratorio. Ma-

De López, L., De Míguez, S. y Marbh, L. (1997). Heavy metals input with phosphate fertilizers used in Argentina. The Science of the Total Environment, 204(3), 245-250. https://doi.org/10.1016/S0048-9697(97)00187-3

Delgadillo, R., Virgen, G., Tabares, S. y Olalde, V. (2001). Bacterias promotoras del crecimiento de plantas: agro-biotecnología. Avance y Perspectiva, 20, 395400. 
Beltrán-Pineda, M. E. y Bernal-Figueroa, A. A. (2022). https://doi.org/10.21789/22561498.1771

Dobereiner, J., Baldani, V. y Baldani, J. (1995). Como isolar e identificar bacterias diazotróficas de plantas não-leguminosas. Embrapa-Spi, Brazil.

El Mujtar, V., Muñoz, N., Mc. Cormick, B., Pulleman, M. y Tittonell, P. (2019). Role and management of soil biodiversity for food security and nutrition; where do we stand? Global Food Security, 20, 132-144. https://doi.org/10.1016/j.gfs.2019.01.007

Himani, M. y Siddhardha, B. (2019). Use of microbial biofertilizers technology in agro-environmental sustainability. En New and Future Developments in Microbial Biotechnology and Bioengineering (pp. 199-211). https://doi.org/10.1016/B978-0-12-818258-1.00013-3

Hurek, T., Reinhold-Hurek, B., Van Montagu, M. y Kellenberger, E. (1994). Root colonization and systemic spreading of Azoarcus sp. Strain BH72 in grasses. Journal of Bacteriology, 176(7), 1913-1923. https://doi.org/10.1128/jb.176.7.19131923.1994

Hynes, R., Craig, K., Covert, D., Smith, R. y Rennie, S. (1995). Liquid rhizobial inoculants for lentil and field pea. J. Prod. Agric., 8(4), 547-552. https://doi.org/10.2134/jpa1995.0547

Kanimozhi, K. y Panneerselvam, A. (2010). Studies on isolation and nitrogen fixation ability of Azospirillum spp.isolated from Thanjavur district. Der Chemica Sinica, 1(3), 138-145.

Kellman, L. y Hillaire-Marcel, C. (2003). Evaluation of nitrogen isotopes as indicators of nitrate contamination sources in an agricultural watershed. Agriculture, Ecosystems and Environment, 95(1), 87-102. https://doi.org/10.1016/S01678809(02)00168-8

Kim, K., Jordan, D. y McDonald, G. (1998). Effect of phosphate solubilizing bacteria and vesicular-arbuscular mycorrhizae on tomato growth and soil microbial activity. Biol. Fertil. Soils, 26, 79-87. https://doi.org/10.1007/s003740050347

Kloepper, J., Lifshitz, R. y Zablotowicz, R. (1989). Free-living bacterial inocula for enhancing crop productivity. Trends Biotechnol., 7(2), 39-44. https://doi.org/10.1016/0167-7799(89)90057-7

Kumar, V. y Narula, N. (1999). Solubilization of inorganic phosphates and growth emergence of wheat as affected by Azotobacter chroococcum mutants. Biol. Fertil. Soils, 28, 301-305. https://doi.org/10.1007/s003740050497

Kundu, M., Mandal, B. y Hazra, G. (2009). Nitrate and fluoride contamination in groundwater of an intensively managed agroecosystem: A functional relationship. Science of the total environment, 407(8), 2771-2782. https://doi.org/10.1016/j.scitotenv.2008.12.048

Lapsansky, E. R., Milroy, A. M., Andales, M. J. y Vivanco, J. M. (2016). Soil memory as a potential mechanism for encouraging sustainable plant health and productivity. Current Opinion in Biotechnology, 38, 137-142. https://doi.org/10.1016/j.copbio.2016.01.014 
Beltrán-Pineda, M. E. y Bernal-Figueroa, A. A. (2022). https://doi.org/10.21789/22561498.1771

Ledesma, C., Bonansea, M., Rodríguez, C. y Sánchez, A. (2013). Determinación de indicadores de eutrofización en el embalse Río Tercero, Córdoba (Argentina). Revista Ciência Agronômica, 44(3), 419-425. http://dx.doi.org/10.1590/S180666902013000300002

Legget, M. y Steckler, S. (2007). Microorganisms mediating an increase in phosphate availability to the plant - How to find and select microorganisms to use in a phosphate inoculant. Segundo curso internacional: "Microorganismos promotores del crecimiento vegetal: rizobacterias y solubilizadores o movilizadores de fosfato". 24-28 de septiembre de 2007, Universidad Nacional de Colombia.

Mehrvarz, S. y Chaichi, M. (2008). Effect of phosphate solubilizing microorganisms and phosphorus chemical fertilizer on forage and grain quality of barely (Hordeum vulgare L.). American-Eurasian J. Agric. \& Environ. Sci., 3(6), 855-860.

Moreno, R., García, T., Storch, J., Muñoz, M., Yáñez, E. y Pérez, E. (2011). Fertilización y corrección edáfica de suelos agrícolas con productos orgánicos. Tecnologi@@ 1 Desarrollo,
https://revistas.uax.es/index.php/tec_des/article/view/571/527

Nautiyal, C. (1999). An efficient microbiological growth medium for screening phosphate-solubilizing microorganisms. FEMS Microbiology Letters, 170(1), 265270. https://doi.org/10.1111/j.1574-6968.1999.tb13383.x

Nziguheba, G. y Smolders, E. (2008). Inputs of trace elements in agricultural soils via phosphate fertilizers in European countries. Sci. Total. Environ., 390(1), 53-57. https://doi.org/10.1016/j.scitotenv.2007.09.031

Pérez, E., Sulbaran, M., Ball, M. Y Yarzabal, L. (2007). Isolation and characterization of mineral phosphate-solubilizing bacteria naturally colonizing a limonitic crust in the south-eastern Venezuelan region. Soil Biology \& Biochemistry, 39(11), 2905-2914. https://doi.org/10.1016/j.soilbio.2007.06.017

Pikovskaya, R. (1948). Mobilization of phosphorus in soil with connection with the vital activity of some microbial species. Mikrobiologiya, 17, 362-370.

Piotrowski, J. \& Rillig, M. (2008). Succession of Arbuscular Mycorrhizal Fungi: Patterns, Causes, and Considerations for Organic Agriculture. Advances in Agronomy, 97, 111-130. https://doi.org/10.1016/S0065-2113(07)00003-X

Riggs, J., Chelius, M., Iniguez, A., Kaeppler, S. Y Triplett, E. (2001). Enhanced maize productivity by inoculation with diazotrophic bacteria. Aust. J. Plant Physiol., 28(9), 829-836. https://doi.org/10.1071/PP01045

Rosas, S., Rovera, M. y Correa, J. (2006). Phosphate-solubilizing Pseudomonas putida can influence the rhizobia-legume symbiosis. Soil Biology \& Biochemistry, 38(12), 3502-3505. https://doi.org/10.1016/j.soilbio.2006.05.008

Sánchez, X., Melgarejo, L., Vanegas, J., Sosa, T. y Fuentes, C. (2006). Diazótrofos de vida libre: perspectivas de aplicación. En L. Castilla y M. Bolaños (Eds.), La biofertilización: una alternativa viable para la nutrición vegetal. Bogotá: Sociedad Colombiana de la Ciencia del Suelo. 
Beltrán-Pineda, M. E. y Bernal-Figueroa, A. A. (2022). https://doi.org/10.21789/22561498.1771

Santillana, N. (2006). Producción de biofertilizantes utilizando Pseudomonas sp. Ecología Aplicada, 5(1,2), 87-91. https://doi.org/10.21704/rea.v5i1-2.322

Shankar, J., Chandra, V. y Singh, D. (2011). Efficient soil microorganisms: A new dimension for sustainable agriculture and environmental development. Agriculture, Ecosystems \& Environment, 140(3-4), 339-353. https://doi.org/10.1016/j.agee.2011.01.017

Smith, R., Alexander, R. y Wolman, M. (1987). Water-quality trends in the
rivers.
nation's
https://doi.org/10.1126/science.235.4796.1607
https://doi.org/10.1126/science.235.4796.1607

Stefan, M., Dunca, S., Olteanu, Z., Oprica, L., Ungureanu, E., Hritcu, L., Mihasan, M. y Cojocaru, D. (2010). Soybean (Glycine max [L] Merr.) Inoculation with Bacillus pumilus RS3 promotes plant growth and increases seed protein yield: relevance for environmentally-friendly agricultural applications. Carpathian Journal of Earth and Environmental Sciences, 5(1), 131-138.

Stephens, J. y Rask, H. (2000). Inoculant production and formulation. Field Crops Research, 65(2-3), 249-258. https://doi.org/10.1016/S0378-4290(99)00090-8

Sttenhoudt, O. y Vanderleyden, J. (2000). Azospirillum, a free living nitrogen-fixing bacterium closely associated with grasses: genetic, biochemical and ecological aspects. FEMS Microbiology Reviews, 24(4), 487-506. https://doi.org/10.1111/j.1574-6976.2000.tb00552.x

Sundara, R. y Sinha, M. (1963). Phosphate dissolving organisms in the soil and the rizosphere. Indian J. Agr. Sci., 33, 272-278.

Takeda, A., Tsukada, H., Takakop, Y., Hisamatsu, S. y Nanzyo, M. (2006). Accumulation of uranium derived from long-term fertilizer applications in a cultivated Andisol. Science of the Total Environment, 367(2-3), 924-931. https://doi.org/10.1016/j.scitotenv.2006.01.006

Tiessen, H. \& Stewart, J. (1985). The biochemistry of soil phosphorus. En D. Caldwell, J. Brierley, y C. Brierley (Eds.), Planetary Ecology. Nueva York: Van Nostraad Reinhold.

Uri, N. (1997). Incorporating the environmental consequences in the fertilizer use decision. Science of the Total Environment, 201(2), 99-111. https://doi.org/10.1016/S0048-9697(97)00088-0

Valero, N. (2003). Potencial biofertilizante de bacterias diazótrofas y solubilizadoras de fosfatos asociadas al cultivo de arroz (Oryza sativa L). [tesis de Maestría en Microbiología]. Bogotá: Universidad Nacional de Colombia.

Verzeaux, J., Hirel, B., Dubois, F., Lea, P. y Tétu, T. (2017). Agricultural practices to improve nitrogen use efficiency through the use of arbuscular mycorrhizae: Basic and agronomic aspects. Plant Science, 264(48-56). https://doi.org/10.1016/j.plantsci.2017.08.004

Vessey, J. (2003). Plant growth promoting rhizobacteria as biofertilizers. Plant and Soil, 255, 571-586. https://doi.org/10.1023/A:1026037216893 
Yanni, G., Rizk, Y., El-Fattah, F., Squartini, A., Corich, V., Giacomini, A., De Bruijn, F., Rademaker, J., Maya-Flores, J., Ostrom, P., Vega-Hernandez, M., Hollingsworth, R., Martínez-Molina, E., Mateos, P., Velazquez, E., Wopereis, J., Triplett, E., Umali-Garcia, M., Anarna, J., Rolfe, B., Ladha, J., Hill. J., Mujoo, J., Perry, K. y Dazzo, F. (2001). The beneficial plant growth-promoting association of Rhizobium leguminosarum bv. Trifolii with rice roots Functional Plant Biology, 28, 845-870. https://doi.org/10.1071/PP01069 